\title{
Physiological shear stress enhances differentiation and mucus-formation of intestinal epithelial cells in vitro.
}

\author{
Marcus Lindner ${ }^{1}$, Anna Laporte ${ }^{2}$, Stephan Block ${ }^{1}$, Laura Elomaa ${ }^{1}$, and Marie Weinhart ${ }^{2}$ \\ ${ }^{1}$ Freie Universitat Berlin \\ ${ }^{2}$ Leibniz Universitat Hannover
}

December 7, 2020

\begin{abstract}
The gastrointestinal (GI) mucus layer plays a pivotal role in tissue homoeostasis and functionality of the gut. However, due to the shortage of affordable, realistic in vitro mucus models, studies with deeper insights into its structure and characteristics are rare. To obtain an improved mucus model, we developed a reusable culture chamber facilitating the application of physiologically relevant GI shear stresses $\left(0.002-0.08 \mathrm{dyn} / \mathrm{cm}^{2}\right)$ to cells in a bioreactor system. Differentiation of a confluent monolayer of human mucus-producing epithelial HT29-MTX cells was monitored under dynamic and static culture conditions. Cells under flow remained highly proliferative and analysis via confocal microscopy revealed superior reorganization into 3-dimensional villi-like structures compared to static culture (up to 120 vs. $80 \mu \mathrm{m}$ in height). Additionally, the median mucus thickness was significantly increased under dynamic conditions compared to static culture ( $41 \pm 14$ vs. $29 \pm 14 \mu \mathrm{m})$ with a simultaneous drastic reduction of culture time from three to two weeks for sufficient maturation into goblet-like cells. We demonstrated the impact of culture conditions on the differentiation of HT29-MTX cells, revealing outstanding in vivo like reorganization of cells and the production of thick adherent mucus networks when cultured under physiological shear stress using our newly designed culture chamber.
\end{abstract}

\section{Introduction}

The luminal surface of a GI tract is a highly dynamic barrier consisting of a constantly renewing epithelial layer covered by mucus (Johansson et al., 2013). For a long time, the mucus was solely attributed to the protection against shear-induced damage and the invasion of pathogens. In recent years this view has changed and it was identified to be in a symbiotic relationship with the commensal microbiome, for example by supporting the growth of indigenous bacteria, which in exchange deliver nutrients to the host (Schroeder, 2019). Additionally, changes in structure and composition of mucus are related to serious illnesses like inflammatory bowel disease, although it is not yet clear whether they are the cause or consequence (Schultsz et al., 1999). Therefore, improved understanding of dynamic mucus architecture is needed.

In general, mucus is a complex biological hydrogel, consisting mostly of water ( $~ 95 \%)$, proteins, salts, nucleic acids and cell debris. Depending on the location within the human GI tract, the thickness of the adherent mucus layer varies between $16 \pm 5 \mu \mathrm{m}$ and $155 \pm 54 \mu \mathrm{m}$ in the duodenum and rectum, respectively (Corfield et al., 2001). Its properties and structure are changing according to variations in $\mathrm{pH}$-value, concentration of calcium ions as well as the action of DNases and pancreatic enzymes (Macierzanka et al., 2014; Müller et al., 2012; Yildiz et al., 2015). The structuring components are mucins, a family of highly O-glycosylated proteins of which 21 members are known up to now. Mucins are divided into two groups: membrane-bound and secreted mucins. The latter are mainly represented by gel-forming mucins, which are able to form complex networks via multimerization through covalent and non-covalent interactions. The main gel-forming mucin in the stomach is MUC5AC, whereas MUC2 is predominant in the intestine (Johansson et al., 2013). To 
study the underestimated influence of mucus in health and disease, there is an urgent need for improved, reproducible cell-based mucus models.

Various mucus models have been published in the past, including ex vivo human and in/ex vivo animal models as well as divergingin vitro models. Even though the in vivo and ex vivo models are physiologically highly relevant due to their high degree of complexity, they suffer from intra- and inter-species variations, limited availability and ethical concerns (Carlson et al., 2018). These restrictions also apply to the use of raw mucus, where not only batch-to-batch variations but also the involved isolation process cause high heterogeneity. Additionally, enzymes in the isolated mucus can cause a degradation and aging over time (Harding, 2006). Albeit the homogeneity of raw mucus can be increased by purification, it is time intensive and results in irreversible alterations of its native structure, composition and physical properties (Groo et al., 2013). Alternatively, defined mucus biosimilars are under development, which is yet complicated by the high complexity of the natural example (Boegh et al., 2014). Instead of using the intricate in vivo models or raw mucus samples, the use of models based on mucus-producing cells circumvents most of the aforementioned limitations. However, the use of primary intestinal cells is restricted by the shortage of donor material as well as their difficult maintenance and short life-time, while the use of stem cell derived intestinal organoids in turn is limited by their hardly accessible internal lumen and structural variabilities as well as the costly supplementation (Kasendra et al., 2018; Wang et al., 2019; Zachos et al., 2016).

Therefore, a lot of intestinal models are based on immortalized cells, most commonly the human colorectal adenocarcinoma cell line Caco-2. It exhibits several properties of enterocytes, including the formation of tight junctions and the expression of drug transporters and metabolic enzymes (Dosh et al., 2018). However, its frequent use for permeability studies suffers from several unphysiological factors, including a too strong barrier function and the lack of mucus production, which in vivo is accomplished by the highly specialized goblet cells. The cell line HT29, originating from a colorectal adenocarcinoma, is one of the most commonly used goblet-like cell lines. It differentiates into a constitutive mucus-forming phenotype in the presence of methotrexate (MTX), with MUC5AC as the main gel-forming mucin produced (Lesuffleur et al., 1990).

Despite the wide use of Caco-2 and HT29 cell cultures as in vitromodels, the thickness and composition of the mucus in these models is rarely well characterized. Since some studies have indicated unphysiologically low mucus thicknesses mostly in the range of a few micrometers (Beduneau et al., 2014; Navabi et al., 2013) an increase in mucus production and thus mucus thickness could further improve the relevance of these models. Previously, the presence of biologically active molecules, such as prostaglandin E2, ATP or vasoactive intestinal peptide as well as bacteria have been detected to induce elevated mucus generation and secretion (Navabi et al., 2013; Sontheimer-Phelps et al., 2020; Wang et al., 2019). Aside from that, the mucus formation can be increased by an underlying substrate or by cultivation under semi-wet or air-liquidinterface conditions (Navabi et al., 2013; Reuter et al., 2018). Furthermore, several groups have reported the shear-induced mucus production of primary cells and Caco-2 cells in an organ-on-a-chip (Chi et al., 2015; Kim et al., 2012; Mahler et al., 2009; Sontheimer-Phelps et al., 2020). Despite all benefits, these models are still limited either by low mucus thicknesses, unphysiological culture conditions, costly supplementation or the requirement for complex equipment.

Therefore, we developed a reusable culture chamber that allows the application of physiological shear stress to cells on conventional microscope slides under laminar flow conditions. We hypothesize that this mechanical influence is sufficient to promote HT29-MTX maturation into goblet-like cells and consequently increases mucus production. Thus, HT29-MTX cells were cultured under defined dynamic conditions and analyzed in terms of proliferation, differentiation and mucus generation. The composition of the adherent mucus layer was further characterized via immunofluorescent staining as well as reverse transcription quantitative PCR (RT-qPCR) and its thickness was determined by confocal microscopy. With the described flow chamber, we aimed to develop an advanced intestinal model with more physiological mucus thickness to enable the better understanding of the structure and composition of mucus under a variety of defined conditions.

\section{Materials and Methods}




\section{Materials}

Dulbecco's Modified Eagle's Medium (DMEM, high glucose, GlutaMAX supplemented), trypsinethylenediamine tetraacetic acid (Trypsin-EDTA), Dulbecco's phosphate-buffered saline (PBS), proliferation assay (Click-iT EdU Cell Proliferation Kit, Alexa Fluor 488), DNA-intercalating dye (Hoechst 33342), proteinase K powder (fromEngyodontium album ), 96 well plates (Black/Clear Bottom), amine-modified fluorescent latex beads (FluoSpheres, $0.2 \mu \mathrm{m}$, yellow-green), MUC5AC-antibody (mouse, monoclonal), cDNA synthesis kit (RevertAid First Strand), qPCR master mix (Maxima ${ }^{\circledR}$ SYBR Green), radioimmunoprecipitation assay buffer (RIPA, Lysis and Extraction Buffer) and protease inhibitor (Halt, EDTA-free) were purchased from Thermo Fisher Scientific (Waltham, MA/USA). Fetal bovine serum (FBS, Superior), nonessential amino acids (MEM-NEAA), octoxynol 9 (Triton X-100), alcian blue solution (1\% in 3\% acetic acid, $\mathrm{pH}$ 2.5), periodic acid Schiff (PAS) staining kit, MUC5B-antibody (rabbit, polyclonal), $p$-nitrophenyl phosphate solution (pNPP, Alkaline Phosphatase Yellow Liquid Substrate) and phalloidin-Atto ${ }^{(\mathrm{r})} 647 \mathrm{~N}$ were obtained from Sigma-Aldrich (St. Louis, MO/USA). Microscope slides (Superfrost ${ }^{(\mathrm{r})}$ ), $4 \%$ paraformaldehyde $\left(\mathrm{PFA}, \mathrm{ROTI}^{(\mathrm{r})}\right.$ Histofix 4\%), tris-(hydroxymethyl)-amino methane (Tris, [?]99,3 \%, PUFFERAN ${ }^{(\mathrm{r})}$ ), EDTA ([?]99), albumin fraction V (BSA, biotin-free) and sodium hydroxide ( $\mathrm{NaOH}$, [?]98 \%) were ordered from Carl Roth (Karlsruhe, Germany). Glacial acetic acid (>99.7) and hydrochloric acid (HCl, 37\%) were purchased from Fisher Scientific (Loughborough, United Kingdom), 6-well tissue culture plates and rectangular cell culture dishes (quadriPERM ${ }^{(\mathrm{r})}$ ) from Sarstedt (Numbrecht, Germany) and syringe filters $\left(\right.$ Minisart $^{(\mathrm{r})}$, PTFE, $0.2 \mu \mathrm{m}$ ) from Sartorius (Göttingen, Germany). MUC1-antibody (rabbit, polyclonal) was obtained from Abcam (Cambridge, United Kingdom), RNA extraction kit (innuPREP RNA Mini Kit 2.0) from AJ Innuscreen (Berlin, Germany), polycarbonate (Makroclear $\left.{ }^{\circledR}\right)$ from Arla Plast (Borensberg, Sweden), mounting medium (ProTaqs ${ }^{\circledR}$ Mount Flour) from Biocyc (Luckenwalde, Germany), DNA-quantitation kit (AccuBlue ${ }^{\circledR}$ Broad Range) from Biotium (Hayward, CA/USA) and antibiotic-antimycotic solution from Biowest (Nuaillé, France). Silicone tubings (Puri-Flex L/S $14^{\circledR}$ ) were purchased from Cole Parmer (Vernon Hills, IL/USA), 12-well cell culture inserts (Transwell ${ }^{\circledR}$, PET, $0.4 \mu \mathrm{m}$ ) from Corning (Corning, NY/USA), HT29-MTX-E12 from ECACC (Porton Down, United Kingdom), thumbscrews (M3x10, DIN 646, stainless steel) from ERIKS Deutschland (Halle, Germany), 3D-printable resin (Dental SG Resin) from Formlabs (Somerville, MA/USA), round coverslips (Ø $18 \mathrm{~mm}$ No.1) from Glaswarenfabrik Karl Hecht (Sondheim v.d. Rhön, Germany), $35 \mathrm{~mm}$ dishes (high) from ibidi (Martinsried, Germany) and glucose/lactate sensors (B.LV5) from Jobst Technologies (Freiburg, Germany). Luer lock to M5 thread adapters (stainless steel) were obtained from Key Surgical (Lensahn, Germany), luer lock to barb connectors (polypropylene) from QOSINA (Ronkonkoma, NY/USA), MUC2-antibody (mouse, monoclonal) from Santa Cruz Biotechnology (Dallas, TX/USA), duplicating silicone (REPLISIL $22 \mathrm{~N}$ ) from SILCONIC ${ }^{\circledR}$ (Lonsee, Germany), 3-[(3-Cholamidopropyl)dimethylammonio]1-propansulfonat (CHAPS) from VWR International (Darmstadt, Germany) and heat-seal sterilization bags (400 x $250 \mathrm{~mm}$ ) from SÜDPACK Medica (Baar, Switzerland).

\section{Chamber design and fabrication}

A cultivation chamber was computer-aided designed (CAD) with Rhinoceros (Version 5.0, Robert McNeel \& Associates, Seattle, WA/USA). The chamber consists of a body which fits and holds a standard 26x76x1 mm microscope slide in its cavity and forms a flow channel of defined height when closed with a lid (Figure 1a). Fluid tight sealing of the chamber was accomplished by insertion of casted gaskets made from medical grade silicone in between body and lid and closing of the chamber with six knurled thumb screws. The applicable wall shear stress $\tau$ was estimated using equation (1)

$\tau=6 \mu X \omega^{-1} h^{-2}(1)$

with the dynamic viscosity $\mu$, the volume flow rate $Q$ as well as the width $w$ and height $h$ of the fluidic channel, which was varied from 0.15 to $6 \mathrm{~mm}$ (Table S1). CAD-files of the resulting chambers and corresponding dimensions can be found in the SI. The chambers were fabricated from bulk polycarbonate by conventional CNC-milling. Homogenizing triangular structures were 3D-printed from a commercially available, biocompatible and autoclavable ink for chambers with a $6 \mathrm{~mm}$ channel height (see SI for detailed information). After fabrication, threads were cut and steel adapters were mounted to connect hoses. All 
materials remained inherently stable after autoclaving for at least 10 cycles.

\section{Computational Fluid Dynamics (CFD)}

The numerical analysis of flow conditions within the designed cultivation chambers was carried out with COMSOL Multiphysics (v5.5 including the CFD-module, COMSOL, Stockholm, Sweden). The inlet flow properties were set to $6.4 \mathrm{~mL} / \mathrm{min}$ and zero pressure was defined as outlet condition. Gravity was included and the dynamic viscosity $\mu$ of the fluid was set to $0.93 \mathrm{cP}$ (Poon, 2020). Meshing was performed using the "physics-controlled"-mesh option as free tetrahedral elements and the mesh-quality was judged based on COMSOL's minimum element quality.

\section{Cell culture}

Human intestinal HT29-MTX cells were cultured in DMEM High Glucose medium, supplemented with $10 \%$ FBS, 1x MEM-NEAA and $1 \mathrm{x}$ antibiotic-antimycotic solution at $37{ }^{\circ} \mathrm{C}$ and $5 \% \mathrm{CO}_{2}$ in a humidified atmosphere. $0.05 \%$ Trypsin-EDTA was used to passage cells once a confluency of $60-80 \%$ was reached.

For static experiments, $2 \times 10^{5}$ cells $/ \mathrm{cm}^{2}$ were seeded in 6 -well plates or on $35 \mathrm{~mm}$ dishes for confocal microscopy. Prior to dynamic cultivation, $2 \times 10^{5}$ cells $/ \mathrm{cm}^{2}$ were seeded on sterilized glass coverslips for thickness determination or microscope slides in rectangular cell culture dishes with the aid of silicone casted cell separators to define a growth area of $2 \times 3 \mathrm{~cm}^{2}$ per slide. Separators were removed $24 \mathrm{~h}$ after cell seeding and cells were cultured for two more days under static conditions to reach confluency.

\section{Bioreactor setup and dynamic cell culture}

The stand-alone bioreactor system (OSPIN, Berlin, Germany) for dynamic culture comprises peristaltic pumps for medium exchange and perfusion, a $\mathrm{pH}$ sensor to monitor and adjust the $\mathrm{pH}$-value as well as temperature control via circulation of heated air. Additionally, a flow cell biosensor for glucose and lactate levels in the medium can be implemented. The mounted peristaltic pumps enable a constant perfusion rate of 2.84 to $14.2 \mathrm{~mL} / \mathrm{min}$ when operated at 20 to $100 \mathrm{RPM}$ with silicone hoses with an inner diameter of 1.6 $\mathrm{mm}$.

For sterilization of all parts, the assembled cultivation circuit was transferred into sterilization pouches and autoclaved for $20 \mathrm{~min}$ at $121{ }^{\circ} \mathrm{C}$ and 2 bar. Afterwards, the reservoir flask of the assembled circuit was filled with fresh medium under sterile conditions and sealed with sterile filters for gas exchange. The circuit was integrated into the bioreactor and equilibrated for $24 \mathrm{~h}$ at a constant fluid flow rate of $6.4 \mathrm{~mL} / \mathrm{min}$ (45 RPM). Subsequently, confluent cells on microscope slides or glass coverslips were transferred into the chamber and cultured under the aforementioned conditions. A complete medium exchange of approximately $40 \mathrm{~mL}$ was performed every $4-5$ days. The applied flow rate generates a physiological surface shear stress of $0.009 \mathrm{dyn} / \mathrm{cm}^{2}$ on the cellular monolayer which is representative for the intestine (Kim et al., 2012). The described conditions are referred to as "dynamic culture conditions".

\section{Cell proliferation assay}

The proliferation of cells was detected via 5-Ethynyl-2'-deoxyuridine (EdU) incorporation according to manufacturer's instructions. Briefly, cells were incubated with $10 \mu \mathrm{M}$ EdU in cell culture medium for $2 \mathrm{~h}$ under static or dynamic conditions. Cells were fixed with $4 \%$ PFA for 10 min, permeabilized with $0.5 \%$ Triton-X 100 for 20 min and afterwards incubated with the EdU-Click-It ${ }^{\mathrm{TM}}$ reaction cocktail for $20 \mathrm{~min}$. Nuclei were counterstained with $1 \mu \mathrm{g} / \mathrm{mL}$ Hoechst 33342 in PBS for 15 min in the dark. Finally, cells were mounted and analyzed via confocal microscopy (LSM800, Carl Zeiss, Jena, Germany). All steps were conducted at room temperature.

\section{Mucus staining and quantification}

To visualize acidic mucins, samples from dynamic and static culture were fixed using PFA, washed with PBS and incubated with Alcian Blue G8x Solution (1\% in 3\% acetic acid) for 15 min at room temperature. Subsequently, the solution was removed and samples were washed with distilled water, followed by washing 
steps with $3 \%$ acetic acid and water to remove residual staining solution. To stain neutral mucins, fixed samples were incubated for 2 min with $0.5 \%$ periodic acid, washed and treated for 10 min with Schiff's reagent, followed by three washing steps with PBS. Stained samples in PBS were imaged using an inverted microscope (Axio Observer Z1, Carl Zeiss, Jena, Germany) equipped with a color camera (Axiocam 105 color, Carl Zeiss, Jena, Germany).

To quantify neutral mucins, cells and adherent mucus were removed from the culture substrate using a cell scraper, vigorously resuspended in PBS $(1 \mathrm{~mL})$, snap frozen in liquid nitrogen and stored at $-80{ }^{\circ} \mathrm{C}$ until further analysis. The cell suspension $(100 \mu \mathrm{L})$ and $2 \% \mathrm{w} / \mathrm{v}$ CHAPS $(100 \mu \mathrm{L})$ were mixed and diluted with PBS $(800 \mu \mathrm{L})$. The resulting suspension was sequentially incubated with periodic acid $(20 \mu \mathrm{L})$ and Schiff's reagent $(100 \mu \mathrm{L})$ for 2 and $1 \mathrm{~h}$, respectively, at $37{ }^{\circ} \mathrm{C}$ in a thermal shaker. The absorbance $\mathrm{A}_{555}$ of the resulting solution $(100 \mu \mathrm{L})$ was measured in 96-well plates using a microplate reader (Infinite M200 PRO, Tecan, Männedorf, Switzerland).

For normalization, DNA content was determined using AccuBlue ${ }^{\circledR}$ assay according to the manufacturer's instructions. In brief, the cell suspension $(100 \mu \mathrm{L})$ was resuspended in $2 \mathrm{x}$ digestion buffer $(20 \mathrm{mM}$ Tris, 2 mM EDTA, $0.2 \%$ Triton X-100 and $1 \mathrm{mg} / \mathrm{mL}$ proteinase $\mathrm{K}$ ) and incubated overnight at $37{ }^{\circ} \mathrm{C}$. AccuBlue ${ }^{\circledR}$ reagent was diluted 1:100 in reaction buffer, mixed with the sample at a ratio of 10:1 and incubated for 15 min in the dark. Samples were excited at $480 \mathrm{~nm}$ and emission $\mathrm{E}_{520}$ was measured in 96-well plates using a microplate plate reader in reference to a standard calibration curve.

\section{Thickness-determination of the adherent mucus layer}

HT29-MTX cells on $18 \mathrm{~mm}$ coverslips were incubated with amine-modified, fluorescent latex beads (Ø 200 $\mathrm{nm}, 1: 500)$ and Hoechst $33342(5 \mu \mathrm{g} / \mathrm{mL})$ in medium for $20 \mathrm{~min}$ under cell culture conditions. Samples were gently washed twice with warm PBS and afterwards analyzed in cell culture medium via confocal microscopy. The Image $J$ based software package FIJI v1.52 (Schindelin et al., 2012) was used for image processing and analysis. Confocal stacks of beads and nuclei were converted from 8-bit grayscale to a binary image via manual thresholding and analyzed with the Particle Analyzer of theBoneJ -plugin v7.0.7 (Doube et al., 2010). All assigned nuclei and beads were allotted to groups according to their $\mathrm{x}$ and $\mathrm{y}$ coordinates in a $15 \times 15$ grid. The differences of z-coordinates (beads minus nuclei) in corresponding groups were calculated to estimate the median thickness ( \pm median absolute deviation, MAD) of the adherent hydrated mucus layer. A minimum of 15 values per sample $(n=5)$ were analyzed to deduce the average mucus layer thickness.

\section{Immunostaining and direct labelling}

Cells were fixed with $4 \%$ PFA for 20 min, permeabilized with $0.1 \%$ Triton X-100 for 10 min, blocked using $5 \%$ BSA in PBS for 90 min and afterwards incubated with primary antibodies against MUC1, MUC2, MUC5AC and MUC5B for 4 hours. Primary antibodies were detected via incubation with fluorophore-coupled secondary antibodies for $90 \mathrm{~min}$ (see Table S2 for further information). All samples were counterstained with Hoechst $33342(10 \mathrm{ng} / \mathrm{mL})$ in PBS and selected samples using phalloidin-Atto ${ }^{\circledR} 647$ for $15 \mathrm{~min}$ in the dark. All steps were performed at room temperature, samples were analyzed via confocal microscopy and images were processed with the Zen Blue software (v2.3).

\section{Gene expression analysis}

Total RNA from HT29-MTX cells was isolated using the innuPREP RNA Mini Kit 2.0 according to the manufacturer's instructions. Isolated RNA $(1 \mu \mathrm{g})$ was reverse transcribed into cDNA using oligo $(\mathrm{dT})_{18}$ and random hexamer primers $(5 \mu \mathrm{mol}$ each). Gene-specific primers were designed with Primer3 https://primer3.ut.ee/ (Untergasser et al., 2012) and used at a concentration of $0.3 \mu \mathrm{M}$. Primer sequences and qPCR conditions can be found in Table S3 and S4. qPCR was performed with the Maxima ${ }^{\circledR}$ SYBR Green qPCR Master Mix by the PikoReal system (Thermo Fisher Scientific, Waltham, MA/USA). Absolute data were normalized to TATA-binding protein (TBP) and RNA Polymerase II Subunit A (POLR2A) and are expressed as fold change compared to Static - Week 1 samples $\left(2^{-\Delta \Delta " \tau}\right.$ method).

\section{Alkaline phosphatase (ALP) activity assay}


To determine intracellular ALP activity, HT29-MTX cells were removed from their substrate using a cell scraper, washed with PBS and centrifuged for $10 \mathrm{~min}$ at 5,000 $\mathrm{x}$ g. PBS was aspirated, cells were resuspended in cold RIPA buffer $(60 \mu \mathrm{L})$, containing 1x EDTA-free protease inhibitor and incubated for 40 min on ice. Reaction tubes were briefly vortexed every $10 \mathrm{~min}$ to ensure complete cell lysis. After incubation, the lysates were centrifuged for $5 \mathrm{~min}$ at maximum speed to sediment insoluble cell debris. The supernatant and ALP working reagent were mixed in a 1:1 ratio in a 96-well plate. The ALP-catalyzed formation of $p$ -nitrophenol was colorimetrically monitored over a period of $60 \mathrm{~min}$ by measuring the change of absorbance as $\Delta\left(\mathrm{A}_{405}-\mathrm{A}_{660}\right)[?] \mathrm{min}^{-1}$ with a microplate reader.

\section{Transepithelial electrical resistance (TEER)}

The electrical resistance of HT29-MTX cells seeded on Transwell ${ }^{\circledR}$ inserts was measured using the Millicell ${ }^{\circledR}$ ERS-2 unit equipped with a STX1 electrode (Millipore, Bedford, MA, USA) to assess their barrier function. To ensure accurate measurements, apical and basolateral medium was replaced by pre-warmed, fresh medium and inserts were kept on a $37{ }^{\circ} \mathrm{C}$ heating plate (HP062, AgnThos, Lidingö, Sweden) for 10 min prior to and during the measurement. Cell culture inserts without cells were measured as reference.

\section{Statistics}

All experiments were conducted at least as triplicates with a minimum of two technical replicates. Statistical significance between two groups was evaluated using the Wilcoxon/Mann-Whitney Test, whereas statistical significance between more than two groups was evaluated using the Kruskal-Wallis test, followed by Dunn's post-hoc test or two-way ANOVA with repeated measurements followed by Tukey's test using OriginPro 2020b (v9.7.5.184, OriginLab, Northampton, MA/USA). Significances are indicated as ${ }^{*} \mathrm{p}[?] \quad 0.05 ;{ }^{* *} \mathrm{p}[?]$ 0.005 and ${ }^{* * *} \mathrm{p}$ [?] 0.0005 .

\section{Results \& Discussion}

\section{Chamber Characterization}

For culture of HT29-MTX cells on standard microscope slides under defined dynamic conditions, an easy to reproduce, reusable flow chamber was designed and manufactured (Figure 1a,b). The geometry of the chamber was shaped to generate homogenous laminar flow on the cell surface with adjustable physiological shear stresses. By varying the height of the flow channel from 0.15 to $6 \mathrm{~mm}$, we mathematically approximated an applicable range of wall shear stresses from 0.004 to $29.7 \mathrm{dyn} / \mathrm{cm} 2$ (Eq. 1), which resembles physiological shear stresses of a variety of human organs and blood vessels (Shemesh et al., 2015). The obtained values were further validated and the fluid flow behavior within the chambers was characterized via CFD-simulations, which revealed differences between simulated and calculated values, with the latter being generally overestimating (Table S1). Chambers with channel heights up to $1.5 \mathrm{~mm}$ already exhibit laminar flow conditions due to a construction-dependent threshold (Figure S1a and S2). As it can be seen from Figure S1b, homogenous laminar flow necessitates the implementation of homogenizing structures (Figure S1c) for chambers with $6 \mathrm{~mm}$ channel height, which were designed on the basis of the conducted CFD simulations. A representative example, including velocity profile and surface shear stress, is shown in Fig 1c and d for a corresponding chamber with $6 \mathrm{~mm}$ channel height and 3D-printed homogenizing triangular features at a volume flow rate of $6.4 \mathrm{~mL} / \mathrm{min}$. The estimated and simulated surface shear stresses were 0.008 and 0.009 $\mathrm{dyn} / \mathrm{cm} 2$, thus matching with physiological shear stresses in the human small intestine (Kim et al., 2012).

Altogether, the design of the chamber allowed an implementation into a stand-alone bioreactor system to cultivate cells under defined dynamic conditions, illustrated in Figure 1e. All materials remained inherently stable after repeated cycles of autoclaving. Additionally, the chamber was operable in a common $\mathrm{CO}_{2}$ incubator with a peristaltic pump (data not shown).

\section{Design of experiments}

The human colon adenocarcinoma-derived cell line HT29-MTX generally requires 21 days to fully differentiate into an intestinal epithelial, mucus-producing cell layer under static conditions (Lesuffleur et al., 1993). 
Their ongoing maturation into functional goblet-like cells is macroscopically visible, since the accumulated mucus layer on top of the cell layer progressively reduces its transparency. It was already shown, that the mucus production of HT29-MTX cells cultured on membranes at the liquid-air interface is enhanced under mechanical stimulation (Navabi et al., 2013; Reuter and Oelschlaeger, 2018). According to Wang et al. (2019), the increased mucus formation and thickness under semi-wet conditions is a result of a reduced dilution of mucin molecules in the absence of apical culture medium. To proof our hypothesis, that the application of physiologically relevant shear stress under laminar conditions is sufficient to accelerate the maturation of HT29-MTX cells and enhance the accompanying mucus production, the impact of static and dynamic culture conditions is compared in this study. Preliminary experiments have shown that the aforementioned visual turbidity of the cultures developed markedly faster and more intense under flow conditions, which suggests a reduced cultivation time of only 14 days under dynamic conditions to yield a mucus-producing epithelial cell layer (data not shown). Hence, the maturation of HT29-MTX cells in terms of proliferation, differentiation and mucus production under static and dynamic conditions was further characterized.

\section{Morphology and proliferation of HT29-MTX cells under static and dynamic conditions}

To monitor differences in the development of integrity and morphology of HT29-MTX cells under static and dynamic culture conditions, the conformation of the cell layer was first monitored at different time points via phase contrast microscopy (Figure 2a). The cell layers were confluent at all times and conditions without any recognizable defects. Additionally, we observed a time-dependent formation of darker regions that are hard to get into focus during microscopic imaging. This phenomenon developed faster and was more pronounced under dynamic conditions in agreement with the macroscopic observation of turbidity when compared to samples from static conditions at the same or even advanced time points. It has already been reported in literature that HT29-MTX monolayers are able to expand 3-dimensionally (Reuter et al., 2018). The presence of multilayered regions, as well as the accumulation of mucus seem to decrease the light transmission in the respective areas and hinder optimal focus adjustment.

To further examine this effect, the influence of culture conditions on the proliferative capacity of HT29MTX cells was detected via incorporation of the thymidine analogue EdU into the newly synthesized DNA of daughter cells and subsequent labelling (Figure 2b). Regardless of their cancerous origin, the proliferative capacity of HT29-MTX cells is known to disappear once cells are reaching confluency (Berger, 2017). However, a larger number of proliferating cells was detected at all analyzed time points under dynamic conditions compared to samples from static culture. These results are consistent with the observed increased proliferation of Caco-2 cells, when cultured under flow conditions (Costello et al., 2017). Furthermore, the simultaneous visualization of nuclei via Hoechst-staining revealed HT29-MTX clusters with a higher fluorescence intensity, particularly at late time points. These clusters share structural similarities with the dark spots on the cell layer detected via phase contrast microscopy and can be attributed to multiple layers of highly proliferative cells and their 3 -dimensional rearrangement beyond cellular monolayers.

\section{Villi formation}

The presence of intestinal micro- and macrovilli and the accompanying increase in overall surface area determines the high absorptive capacity of the small intestine (Helander and Fandriks, 2014). Based on the previous data on morphology and proliferation, we further examined the cellular reorganization via visualization of the cytoskeleton. The 3-dimensional expansion of HT29-MTX cells under static and dynamic conditions can be seen in Figure 3a. For both culture conditions, actin-enclosed empty rounded structures are visible in the lower regions of the epithelial layer, which are covered by cells on the top. In literature, such structures within the HT29-MTX cell layer were previously described to be mucus-containing vacuoles without attribution of a function (Navabi et al., 2013; Reuter et al., 2018). In our hands, these structures did not contain mucins, but increased over time in number and size and merged to form larger entities which are overgrown by cell layers, resulting in a 3-dimensional expansion of the cellular monolayer. These defined villi-like structures reached up to $80 \mu \mathrm{m}$ in height after three weeks of static and $120 \mu \mathrm{m}$ after two weeks of dynamic culture, validating the faster and more sufficient maturation and reorganization of the cellular 
layer under flow. This restructuring and expansion are associated with an increased surface area, illustrated in Figure 3b. In addition to the already described rounded structures at the bottom of the cellular layer, we detected detached apically located spheroids containing highly MUC2-producing cells (Figure 3a (white arrow) and Figure S3).

\section{Mucus characterization}

Mucus synthesis and secretion, a major function of the intestinal epithelium (Johansson et al., 2013), was examined for both culture conditions. Staining of acidic and neutral mucins revealed a time-dependent and ongoing increase of the respective mucins under static and dynamic culture conditions (Figure $4 \mathrm{a}, \mathrm{b}$ ). The staining intensified more rapidly under dynamic conditions and both acidic and neutral mucins seem to be located preferentially close to the aforementioned villi structures, which becomes most obvious under static conditions. Because the presence of multicellular layers is also accompanied by a reduction of visible light transparency the color images of the stained samples might misleadingly suggest higher amounts of mucins at cell dense positions with 3-dimensional villi structures. Thus, we additionally determined the amount of neutral mucins per cell and were able to confirm the time-dependent increase in mucus production under both culture conditions (Figure 4c). Compared to the static conditions after week 1, the secreted mucin amount significantly increased up to 2 -fold or 2.4 -fold in three weeks under static or in two weeks under dynamic culture conditions, respectively. These results clearly demonstrate the accelerated differentiation of HT29-MTX cells into goblet-like mucus-producing cells.

Adherent mucus is commonly analyzed after fixation of the respective samples, causing the lack of data on mucus characteristics under native conditions. Unfortunately, common fixation methods lead to dehydration and thus a collapse of the adherent mucus, which does not represent its native structure. Consequently, the conventional fixation decreases the mucus thickness, resulting in thickness values in the range of a few micrometers for fixed HT29-MTX cells (Beduneau et al., 2014; Navabi et al., 2013; Reuter et al., 2018). Even though there are some fixatives used that supposedly preserve its native structure, this is mutually dependent on the sample origin as the adherent mucus may be altered by multiple washing steps and further processing (Rohe et al., 2018). To overcome this obstacle, we incubated the native cultures with amine-modified fluorescent beads, which stick to the charged mucus via electrostatic interactions, and counterstained the cell nuclei with Hoechst. Confocal imaging and further image processing of z-stacks allowed to measure the average distance between nuclei and beads sticking to the mucus surface (Figure 4d). We observed native mucus thicknesses reaching up to $83 \mu \mathrm{m}$ after three weeks under static and $148 \mu \mathrm{m}$ after only two weeks under dynamic conditions. Thus, cultivation under physiological shear stress yielded an increase in maximum mucus thickness by $78 \%$. The corresponding averaged thicknesses were $29 \pm 14 \mu \mathrm{m}$ and $41 \pm 14 \mu \mathrm{m}$, respectively, indicating a significant average mucus thickness increase of $42 \%$ for flow conditions. Furthermore, the obtained number of analyzable data points per condition provides indication of the mucus integrity. As a correlating set of data points was only valid if nuclei as well as beads sticking to the mucus surface were present, the 2.1-fold increase in available correlating data points under dynamic compared to static conditions (299 vs. 139) indicates an improved mucus coverage of the cell layer after application of shear stress.

\section{Immunofluorescent staining of selected mucins}

To investigate the distribution and localization of selected mucins, the best characterized transmembrane mucin MUC1 and the two main GI secreted mucins MUC2 and MUC5AC were visualized via immunofluorescence (Figure 5). In general, all examined mucins were expressed at higher levels after cultivation of the cells under dynamic culture conditions and they were mainly located at the tips of the 3-dimensional villi-like structures, as it can be concluded from the images showing the counterstaining of the nuclei. An enhanced shear-induced polarization was shown before for various cell types, including epithelial and endothelial cells of different origins (Navabi et al., 2013; Wojciak-Stothard and Ridley, 2003). Furthermore, mucins are known to protect the underlying epithelium from mechanical stress (Johansson et al., 2013), which is expected to be highest at the tip of the villi-like structures under dynamic conditions. MUC1 was hardly detectable under static conditions, whereas the tips of the villi-like structures of dynamically cultured cells were api- 
cally covered by MUC1. The stress-induced production of MUC2 was shown before for HT29-MTX cells (Reuter et al., 2018) and also for Caco-2 cells, which usually do not express MUC2 (Kim and Ingber, 2013). In our experiments, MUC2 seemed to be mainly stored intracellularly in the aforementioned actin-enclosed spheroids (Figure 5 and S3). MUC5AC is known to be highly expressed in the stomach and the respiratory tract (Johansson and Hansson, 2016) and it is the main secreted mucin of HT29-MTX cells (Lesuffleur et al., 1993). It can multimerize by forming disulfide bonds and contributes to the formation of a dense mucus network (Sheehan et al., 2004). As it can be seen in Figure 5, under dynamic culture conditions the secreted MUC5AC is forming a heavily branched network, which seems to span the tips of the villi-structures and as such may contribute to an increased robustness of the formed mucus layer. The immunofluorescent staining further supports the observation of an increased mucus coverage of the cellular layer after cultivation under flow conditions. Additionally, the increased generation of secreted mucins after cultivation of the cells for only two weeks under dynamic conditions compared to a cultivation for three weeks under static conditions confirms the accelerated maturation of the HT29-MTX cells in direction of the goblet cell phenotype. Further immunofluorescent staining for MUC5B as well as images from various time points can be found in Figure S4.

Since the immunofluorescent staining of MUC5AC showed high similarities to mucus networks found on the surface of airway epithelia, previously reported by Sheehan et al. (2000), we conducted a co-staining of MUC5AC and the main secreted airway mucin 5B (Figure 6). Interestingly, both mucins were colocalized in the previously observed branched networks and, to a lesser extent, in secretory vesicles as indicated by the white arrow. Imaging at higher magnifications revealed diameters for these single fibers as small as 200 nm. A similar colocalization of fibrous MUC5AC and MUC5B in mucus networks is commonly observed in lung-derived mucus samples (Fischer et al., 2019). To the best of our knowledge, our results are first to show a fibrous and heavily entangled network of MUC5AC and MUC5B strands produced and secreted by the intestinal HT29-MTX cell line.

\section{Gene expression analysis}

We further examined the influence of culture conditions on the transcriptional levels of selected genes, including markers for the cellular state or differentiation, as well as transmembrane and secreted mucin genes. mRNA levels of markers for the cellular state and differentiation are shown in Figure 7a. GAPDH, a frequently used house keeper gene in $\mathrm{qPCR}$, was herein chosen as an indicator for the metabolic activity of cells and is closely related to cellular proliferation (Meyer-Siegler et al., 1992). As it is an enzyme of glycolysis, the increased rates of glucose consumption for dynamically cultured cells, which are reported in literature (Vinci et al., 2010), could also result in higher GAPDH expression. We indeed observed an increase in GAPDH mRNA-levels over time, which was highly elevated under dynamic culture conditions, compared to static conditions. This observation additionally highlights the importance of a conscientious choice of reference genes for mRNA quantification. Since the increased expression of mucins indicated an increased differentiation of HT29-MTX into goblet-like cells, we determined the mRNA levels of the enterocyte marker intestinal alkaline phosphatase (ALPi, Figure 7a). Under static culture conditions the levels remained unaltered, whereas after two weeks of dynamic culture a significant reduction of ALPi mRNAlevels was detectable. This decrease was confirmed on the protein level (Figure 7b), with intracellular enzyme activity being reduced already after one week of dynamic culture. The initial discrepancy between mRNA and intracellular protein level could be explained by an increased secretion of the enzyme under dynamic conditions.

Next, we investigated the mRNA levels of the transmembrane mucins MUC1, MUC4, MUC12 and MUC17 (Figure 7c) and observed an increase of all expression levels after one week of dynamic cultivation. These mucins are known to play an essential role in protecting the epithelium from surface shear stress (Johansson et al., 2013; van Putten and Strijbis, 2017). Additionally, transmembrane mucins are involved in intercellular adhesion processes and may be necessary for the observed rearrangement of the cells and cytoskeleton into villi-like structures, which is much more pronounced under dynamic culture conditions. The MUC12 and MUC17 mRNA levels decreased after two weeks of dynamic culture, which might be the result of an ongoing 
adaption of the cells to the applied shear stress. In contrast, no decrease in MUC1 and MUC4 mRNA levels was observed. It is known from literature, that these two mucins are promoting cellular proliferation (Albrecht and Carraway, 2011), and a constantly high expression is thus in line with the detected high levels of proliferation under dynamic conditions (Figure 2). mRNA levels of the transmembrane mucin MUC3 (Figure 7c), which is reported to be highly expressed in HT29-MTX cells (Lesuffleur et al., 1993), significantly decreased under dynamic conditions. This mucin is also known to be mainly expressed in enterocytes (van Klinken et al., 1996). Thus, the decrease of MUC3 mRNA levels under dynamic conditions is consistent with our data on ALPi expression and activity levels.

The expression of secreted mucins is known to be regulated to maintain a constant thickness of the adherent mucus layer (Strous and Dekker, 1992). The mRNA levels of MUC2, MUC5AC and MUC5B are shown in Figure 7d. Their main function is the protection of the underlying epithelium. It is reported that the expression of MUC2 is stress-induced (Kim et al., 2017), which could be an explanation for the observed trend of higher mRNA levels after one week of dynamic culture. However, these changes in gene expression were not significant. It was already reported in literature that mRNA levels of MUC5AC do not necessarily correlate with protein levels detected by immunohistochemistry, suggesting other regulation mechanisms on the translational than on the transcriptional level (Lievin-Le Moal et al., 2002). We also observed this discrepancy between the transcriptional and translational level for MUC5AC, since after an increase in expression after one week of dynamic culture, the mRNA-level was reduced after two weeks under flow conditions, whereas immunofluorescent microscopy images revealed a marked increase of secreted MUC5AC protein from dynamic culture week one to two (Figure S4). Reasons might be a delay between production and secretion of mucins and the massive difference in volume ( $>1.000$ fold) between their stored and secreted form (Birchenough et al., 2015). In general, data on MUC5B produced by HT29-MTX cells are rare. Smirnova et al. (2003) reported an increase of MUC5AC and MUC5B mRNA and secreted protein after stimulation of HT29-MTX cells with bacterial lipopolysaccharides, which again shows the protective role of these mucins for the cellular layer. We observed a smaller absolute change in mRNA levels for MUC5B compared to MUC5AC but a similar tendency with a decrease in their mRNA levels after two weeks of dynamic culture, which could again be due to an adaption of the cells to the shear stress or due to sufficient mucus coverage of the cellular layer (Strous and Dekker, 1992). The similar pattern of MUC5AC and MUC5B gene expression suggests a common regulatory mechanism for both mucins (Wan et al., 2014), which would be in line with the co-storage of these mucins in secretory vesicles shown above (Figure 6).

\section{Epithelial barrier function}

For a characterization of the epithelial barrier function of differently cultured HT29-MTX monolayers, we aimed at TEER measurements. Therefore, a confluent monolayer has to be grown on a porous membrane of a cell culture insert. In order to transfer the established well-defined dynamic cell culture conditions for the fast maturation and differentiation of HT29-MTX cells from the microscopic slides to commercial cell culture inserts, we developed a fully 3D-printed culture chamber that enables the culture of cells on conventional inserts under flow conditions. To make cells on inserts accessible to shear stress in the chamber, they have to be seeded in a reverse manner onto the bottom of the insert membrane, as visualized in Figure 8a. In contrast to Caco-2 cells, which are the most commonly used model to mimic the intestinal barrier, HT29-MTX cells only form weak barriers as indicated by low TEER values (Schimpel et al., 2014). Beduneau et al. (2014) reported a time-dependent barrier formation of HT29-MTX cells developing from approximately 20 to 60 $\Omega^{*} \mathrm{~cm}^{2}$ from day 7 to 14 in static culture. These values were comparable to our TEER-measurements of static cultures with $11.3 \pm 4.0$ and $40.5 \pm 3.8 \Omega^{*} \mathrm{~cm}^{2}$, respectively (Figure 8b). Notably, an additional increase in barrier function of HT29-MTX cell layers under reverse static conditions was observed, reaching $56.3 \pm$ $3.6 \Omega^{*} \mathrm{~cm}^{2}$ after two weeks. It is reported with Caco-2 cells, that static, reverse, upside-down cultivation on inserts had a qualitatively similar effect on differentiation and cellular reorganization as the application of fluid flow (Shen et al., 2014). Moreover, upon application of defined shear stress of $0.012 \mathrm{dyn} / \mathrm{cm}^{2}$ the TEER value of reversely cultured HT29-MTX cell layers was further enhanced compared to static cultures resulting in $37.3 \pm 4.3 \Omega^{*} \mathrm{~cm}^{2}$ after one week followed by a more than 2-fold increase of the value, reaching $87.6 \pm 4.2 \Omega^{*} \mathrm{~cm}^{2}$ after two weeks of dynamic culture (Figure 8b). A possible explanation for this drastic 
raise of the TEER value might be the increase in thickness of the cellular layer due to the applied shear stress, which consequently affects the electrical resistance. The former detected villi-like structures similarly developed also on porous substrates under the dynamic conditions (data not shown).

\section{Conclusion}

A reusable and sterilizable flow chamber was designed to apply physiologically relevant mechanical stress to HT29-MTX cells seeded on conventional microscopy slides. These conditions improved the reorganization of the cellular layer to resemble more closely the physiological intestinal shape with an increased surface area via villi formation. Additionally, the required culture time for sufficient maturation was drastically reduced and a thick mucus layer could be detected adherent to the cells. Analysis of the mucus on the transcriptional and translational level revealed heavily branched networks of various mucins. We were able to study the mucus barrier in detail and demonstrated the high impact of the application of physiological shear stresses as low as $0.009 \mathrm{dyn} / \mathrm{cm}^{2}$ on cellular differentiation, mucus production and 3D-reorganization of the cellular layer without further need for expensive culture supplements or equipment. In the future, by transferring this technique to the cultivation of intestinal monolayers derived from organoids or stem cells, their physiological behavior in terms of villi formation and especially mucus production could be enhanced. Therefore, the obtained results are an important contribution for the future development of advanced mucus models to mimic the intestinal barrier in vitro .

\section{Acknowledgments}

The authors acknowledge the financial support from HZG Graduate School of Macromolecular Bioscience and the collaborative research center SFB 1449 funded by the Deutsche Forschungsgemeinschaft.

\section{Author Disclosure Statement}

Declarations of interest: none

\section{Author Contributions}

M.L. and M.W. designed the study. S.B. initiated the native mucus thickness measurement. M.L. and L.E. performed the experiments and all authors contributed to data analysis. A.L. and M.L. wrote the manuscript in consultation with M.W. and L.E.. All authors have read and agreed to the published version of the manuscript.

Albrecht, H., \& Carraway, K. L., 3rd. (2011). MUC1 and MUC4: switching the emphasis from large to small. Cancer Biotherapy and Radiopharmaceuticals, 26 (3), 261-271. https://doi.org/10.1089/cbr.2011.1017

Beduneau, A., Tempesta, C., Fimbel, S., Pellequer, Y., Jannin, V., Demarne, F., \& Lamprecht, A. (2014). A tunable Caco-2/HT29-MTX co-culture model mimicking variable permeabilities of the human intestine obtained by an original seeding procedure. European Journal of Pharmaceutics and Biopharmaceutics, 87 (2), 290-298. https://doi.org/10.1016/j.ejpb.2014.03.017

Birchenough, G. M., Johansson, M. E., Gustafsson, J. K., Bergstrom, J. H., \& Hansson, G. C. (2015). New developments in goblet cell mucus secretion and function. Mucosal Immunology, 8 (4), 712-719. https://doi.org/10.1038/mi.2015.32

Boegh, M., Baldursdottir, S. G., Mullertz, A., \& Nielsen, H. M. (2014). Property profiling of biosimilar mucus in a novel mucus-containing in vitro model for assessment of intestinal drug absorption. European Journal of Pharmaceutics and Biopharmaceutics, 87 (2), 227-235. https://doi.org/10.1016/j.ejpb.2014.01.001

Carlson, T. L., Lock, J. Y., \& Carrier, R. L. (2018). Engineering the Mucus Barrier. Annual Review of Biomedical Engineering, 20 , 197-220. https://doi.org/10.1146/annurev-bioeng-062117-121156

Chi, M., Yi, B., Oh, S., Park, D. J., Sung, J. H., \& Park, S. (2015). A microfluidic cell culture device (muFCCD) to culture epithelial cells with physiological and morphological properties that mimic those of the human intestine. Biomedical Microdevices, 17 (3), 9966. https://doi.org/10.1007/s10544-015-9966-5 
Corfield, A. P., Carroll, D., Myerscough, N., \& Probert, C. S. (2001). Mucins in the gastrointestinal tract in health and disease.Frontiers in Bioscience, 6 (3), D1321-1357. https://doi.org/10.2741/corfield

Costello, C. M., Phillipsen, M. B., Hartmanis, L. M., Kwasnica, M. A., Chen, V., Hackam, D., Chang, M. W., Bentley, W. E., \& March, J. C. (2017). Microscale Bioreactors for in situ characterization of GI epithelial cell physiology. Scientific Reports, 7 (1), 12515. https://doi.org/10.1038/s41598-017-12984-2

Dosh, R. H., Jordan-Mahy, N., Sammon, C., \& Le Maitre, C. L. (2018). Tissue Engineering Laboratory Models of the Small Intestine.Tissue Engineering Part B: Reviews, 24 (2), 98-111. https://doi.org/10.1089/ten.teb.2017.0276

Doube, M., Klosowski, M. M., Arganda-Carreras, I., Cordelieres, F. P., Dougherty, R. P., Jackson, J. S., Schmid, B., Hutchinson, J. R., \& Shefelbine, S. J. (2010). BoneJ: Free and extensible bone image analysis in ImageJ. Bone, 47 (6), 1076-1079. https://doi.org/10.1016/j.bone.2010.08.023

Fischer, A. J., Pino-Argumedo, M. I., Hilkin, B. M., Shanrock, C. R., Gansemer, N. D., Chaly, A. L., Zarei, K., Allen, P. D., Ostedgaard, L. S., Hoffman, E. A., Stoltz, D. A., Welsh, M. J., \& Abou Alaiwa, M. H. (2019). Mucus strands from submucosal glands initiate mucociliary transport of large particles. JCI Insight, 4 (1). https://doi.org/10.1172/jci.insight.124863

Groo, A. C., Saulnier, P., Gimel, J. C., Gravier, J., Ailhas, C., Benoit, J. P., \& Lagarce, F. (2013). Fate of paclitaxel lipid nanocapsules in intestinal mucus in view of their oral delivery.International Journal of Nanomedicine, 8 , 4291-4302. https://doi.org/10.2147/IJN.S51837

Harding, S. E. (2006). Trends in muco-adhesive analysis. Trends in Food Science 83 Technology, 17 (5), 255-262. https://doi.org/10.1016/j.tifs.2005.12.007

Helander, H. F., \& Fandriks, L. (2014). Surface area of the digestive tract - revisited. Scandinavian Journal of Gastroenterology, 49 (6), 681-689. https://doi.org/10.3109/00365521.2014.898326

Johansson, M. E., \& Hansson, G. C. (2016). Immunological aspects of intestinal mucus and mucins. Nature Reviews: Immunology, 16 (10), 639-649. https://doi.org/10.1038/nri.2016.88

Johansson, M. E., Sjovall, H., \& Hansson, G. C. (2013). The gastrointestinal mucus system in health and disease. Nature Reviews: Gastroenterology $\&$ Hepatology, 10 (6), 352-361. https://doi.org/10.1038/nrgastro.2013.35

Kasendra, M., Tovaglieri, A., Sontheimer-Phelps, A., Jalili-Firoozinezhad, S., Bein, A., Chalkiadaki, A., Scholl, W., Zhang, C., Rickner, H., Richmond, C. A., Li, H., Breault, D. T., \& Ingber, D. E. (2018). Development of a primary human Small Intestine-on-a-Chip using biopsy-derived organoids. Scientific Reports, 8 (1), 2871. https://doi.org/10.1038/s41598-018-21201-7

Kim, H. J., Huh, D., Hamilton, G., \& Ingber, D. E. (2012). Human gut-on-a-chip inhabited by microbial flora that experiences intestinal peristalsis-like motions and flow. Lab on a Chip, 12 (12), 2165-2174. https://doi.org/10.1039/c2lc40074j

Kim, H. J., \& Ingber, D. E. (2013). Gut-on-a-Chip microenvironment induces human intestinal cells to undergo villus differentiation. Integrative Biology: Quantitative Biosciences from Nano to Macro, 5 (9), 1130-1140. https://doi.org/10.1039/c3ib40126j

Kim, S. W., Ehrman, J., Ahn, M. R., Kondo, J., Lopez, A. A. M., Oh, Y. S., Kim, X. H., Crawley, S. W., Goldenring, J. R., Tyska, M. J., Rericha, E. C., \& Lau, K. S. (2017). Shear stress induces noncanonical autophagy in intestinal epithelial monolayers. Molecular Biology of the Cell, 28 (22), 3043-3056. https://doi.org/10.1091/mbc.E17-01-0021

Lesuffleur, T., Barbat, A., Dussaulx, E., \& Zweibaum, A. (1990). Growth adaptation to methotrexate of HT-29 human colon carcinoma cells is associated with their ability to differentiate into columnar absorptive and mucus-secreting cells. Cancer Research, 50 (19), 6334-6343. 
Lesuffleur, T., Porchet, N., Aubert, J. P., Swallow, D., Gum, J. R., Kim, Y. S., Real, F. X., \& Zweibaum, A. (1993). Differential expression of the human mucin genes MUC1 to MUC5 in relation to growth and differentiation of different mucus-secreting HT-29 cell subpopulations.Journal of Cell Science, 106 ( Pt 3) , 771-783.

Lievin-Le Moal, V., Huet, G., Aubert, J. P., Bara, J., Forgue-Lafitte, M. E., Servin, A. L., \& Coconnier, M. H. (2002). Activation of mucin exocytosis and upregulation of MUC genes in polarized human intestinal mucin-secreting cells by the thiol-activated exotoxin listeriolysin O.Cellular Microbiology, 4 (8), 515-529. https://doi.org/10.1046/j.1462-5822.2002.00210.x

Macierzanka, A., Mackie, A. R., Bajka, B. H., Rigby, N. M., Nau, F., \& Dupont, D. (2014). Transport of particles in intestinal mucus under simulated infant and adult physiological conditions: impact of mucus structure and extracellular DNA. PloS One, 9 (4), e95274. https://doi.org/10.1371/journal.pone.0095274

Mahler, G. J., Esch, M. B., Glahn, R. P., \& Shuler, M. L. (2009). Characterization of a gastrointestinal tract microscale cell culture analog used to predict drug toxicity. Biotechnology and Bioengineering, 104 (1), 193-205. https://doi.org/10.1002/bit.22366

Meyer-Siegler, K., Rahman-Mansur, N., Wurzer, J. C., \& Sirover, M. A. (1992). Proliferative dependent regulation of the glyceraldehyde-3-phosphate dehydrogenase/uracil DNA glycosylase gene in human cells. Carcinogenesis, 13 (11), 2127-2132. https://doi.org/10.1093/carcin/13.11.2127

Muller, C., Leithner, K., Hauptstein, S., Hintzen, F., Salvenmoser, W., \& Bernkop-Schnurch, A. (2012). Preparation and characterization of mucus-penetrating papain/poly(acrylic acid) nanoparticles for oral drug delivery applications. Journal of Nanoparticle Research, 15 (1), 1353. https://doi.org/10.1007/s11051-012$1353-\mathrm{z}$

Navabi, N., McGuckin, M. A., \& Linden, S. K. (2013). Gastrointestinal cell lines form polarized epithelia with an adherent mucus layer when cultured in semi-wet interfaces with mechanical stimulation. PloS One, 8 (7), e68761. https://doi.org/10.1371/journal.pone.0068761

Poon, C. (2020). Measuring the density and viscosity of culture media for optimized computational fluid dynamics analysis of in vitrodevices. bioRxiv , 2020.2008.2025.266221. https://doi.org/10.1101/2020.08.25.266221

Reuter, C., Alzheimer, M., Walles, H., \& Oelschlaeger, T. A. (2018). An adherent mucus layer attenuates the genotoxic effect of colibactin.Cellular Microbiology, 20 (2), e12812-e12812. https://doi.org/10.1111/cmi.12812

Reuter, C., \& Oelschlaeger, T. A. (2018). Enhancement of Mucus Production in Eukaryotic Cells and Quantification of Adherent Mucus by ELISA. Bio-Protocol, 8 (12). https://doi.org/10.21769/BioProtoc.2879

Rohe, I., Huttner, F. J., Plendl, J., Drewes, B., \& Zentek, J. (2018). Comparison of different histological protocols for the preservation and quantification of the intestinal mucus layer in pigs. European Journal of Histochemistry, 62 (1), 2874. https://doi.org/10.4081/ejh.2018.2874

Schimpel, C., Teubl, B., Absenger, M., Meindl, C., Frohlich, E., Leitinger, G., Zimmer, A., \& Roblegg, E. (2014). Development of an advanced intestinal in vitro triple culture permeability model to study transport of nanoparticles. Molecular Pharmaceutics, 11 (3), 808-818. https://doi.org/10.1021/mp400507g

Schindelin, J., Arganda-Carreras, I., Frise, E., Kaynig, V., Longair, M., Pietzsch, T., Preibisch, S., Rueden, C., Saalfeld, S., Schmid, B., Tinevez, J. Y., White, D. J., Hartenstein, V., Eliceiri, K., Tomancak, P., \& Cardona, A. (2012). Fiji: an open-source platform for biological-image analysis. Nature Methods, 9 (7), 676-682. https://doi.org/10.1038/nmeth.2019

Schroeder, B. O. (2019). Fight them or feed them: how the intestinal mucus layer manages the gut microbiota. Gastroenterology Report, 7 (1), 3-12. https://doi.org/10.1093/gastro/goy052 
Schultsz, C., Van Den Berg, F. M., Ten Kate, F. W., Tytgat, G. N., \& Dankert, J. (1999). The intestinal mucus layer from patients with inflammatory bowel disease harbors high numbers of bacteria compared with controls. Gastroenterology, 117 (5), 1089-1097. https://doi.org/10.1016/s0016-5085(99)70393-8

Sheehan, J. K., Brazeau, C., Kutay, S., Pigeon, H., Kirkham, S., Howard, M., \& Thornton, D. J. (2000). Physical characterization of the MUC5AC mucin: a highly oligomeric glycoprotein whether isolated from cell culture or in vivo from respiratory mucous secretions. Biochemical Journal, 347 Pt 1 , 37-44.

Sheehan, J. K., Kirkham, S., Howard, M., Woodman, P., Kutay, S., Brazeau, C., Buckley, J., \& Thornton, D. J. (2004). Identification of molecular intermediates in the assembly pathway of the MUC5AC mucin. Journal of Biological Chemistry, 279 (15), 15698-15705. https://doi.org/10.1074/jbc.M313241200

Shemesh, J., Jalilian, I., Shi, A., Heng Yeoh, G., Knothe Tate, M. L., \& Ebrahimi Warkiani, M. (2015). Flow-induced stress on adherent cells in microfluidic devices. Lab on a Chip, 15 (21), 4114-4127. https://doi.org/10.1039/c5lc00633c

Shen, C., Meng, Q., \& Zhang, G. (2014). Design of 3D printed insert for hanging culture of Caco-2 cells. Biofabrication, 7 (1), 015003. https://doi.org/10.1088/1758-5090/7/1/015003

Smirnova, M. G., Guo, L., Birchall, J. P., \& Pearson, J. P. (2003). LPS up-regulates mucin and cytokine mRNA expression and stimulates mucin and cytokine secretion in goblet cells. Cellular Immunology, 221 (1), 42-49. https://doi.org/10.1016/s0008-8749(03)00059-5

Sontheimer-Phelps, A., Chou, D. B., Tovaglieri, A., Ferrante, T. C., Duckworth, T., Fadel, C., Frismantas, V., Sutherland, A. D., Jalili-Firoozinezhad, S., Kasendra, M., Stas, E., Weaver, J. C., Richmond, C. A., Levy, O., Prantil-Baun, R., Breault, D. T., \& Ingber, D. E. (2020). Human Colon-on-a-Chip Enables Continuous In Vitro Analysis of Colon Mucus Layer Accumulation and Physiology. Cellular and Molecular Gastroenterology and Hepatology, 9 (3), 507-526. https://doi.org/10.1016/j.jcmgh.2019.11.008

Strous, G. J., \& Dekker, J. (1992). Mucin-type glycoproteins. Critical Reviews in Biochemistry and Molecular Biology, 27 (1-2), 57-92. https://doi.org/10.3109/10409239209082559

Untergasser, A., Cutcutache, I., Koressaar, T., Ye, J., Faircloth, B. C., Remm, M., \& Rozen, S. G. (2012). Primer3-new capabilities and interfaces. Nucleic Acids Research, 40 (15), e115. https://doi.org/10.1093/nar/gks596

van Klinken, B. J., Oussoren, E., Weenink, J. J., Strous, G. J., Buller, H. A., Dekker, J., \& Einerhand, A. W. (1996). The human intestinal cell lines Caco-2 and LS174T as models to study cell-type specific mucin expression. Glycoconjugate Journal, 13 (5), 757-768. https://doi.org/10.1007/BF00702340

van Putten, J. P. M., \& Strijbis, K. (2017). Transmembrane Mucins: Signaling Receptors at the Intersection of Inflammation and Cancer. Journal of Innate Immunity, 9 (3), 281-299. https://doi.org/10.1159/000453594

Vinci, B., Murphy, E., Iori, E., Marescotti, M. C., Avogaro, A., \& Ahluwalia, A. (2010). Flow-regulated glucose and lipid metabolism in adipose tissue, endothelial cell and hepatocyte cultures in a modular bioreactor. Biotechnology Journal, 5 (6), 618-626. https://doi.org/10.1002/biot.201000009

Wan, L. Y., Allen, K. J., Turner, P. C., \& El-Nezami, H. (2014). Modulation of mucin mRNA (MUC5AC and MUC5B) expression and protein production and secretion in Caco-2/HT29-MTX co-cultures following exposure to individual and combined Fusarium mycotoxins.Toxicological Sciences, 139 (1), 83-98. https://doi.org/10.1093/toxsci/kfu019

Wang, Y., Kim, R., Sims, C. E., \& Allbritton, N. L. (2019). Building a Thick Mucus Hydrogel Layer to Improve the Physiological Relevance of In Vitro Primary Colonic Epithelial Models. Cellular and Molecular Gastroenterology and Hepatology, 8 (4), 653-655 e655. https://doi.org/10.1016/j.jcmgh.2019.07.009

Wojciak-Stothard, B., \& Ridley, A. J. (2003). Shear stress-induced endothelial cell polarization is mediated by Rho and Rac but not Cdc42 or PI 3-kinases. Journal of Cell Biology, 161 (2), 429-439. 
https://doi.org/10.1083/jcb.200210135

Yildiz, H. M., Speciner, L., Ozdemir, C., Cohen, D. E., \& Carrier, R. L. (2015). Foodassociated stimuli enhance barrier properties of gastrointestinal mucus. Biomaterials, 54 , 1-8. https://doi.org/10.1016/j.biomaterials.2015.02.118

Zachos, N. C., Kovbasnjuk, O., Foulke-Abel, J., In, J., Blutt, S. E., de Jonge, H. R., Estes, M. K., \& Donowitz, M. (2016). Human Enteroids/Colonoids and Intestinal Organoids Functionally Recapitulate Normal Intestinal Physiology and Pathophysiology. Journal of Biological Chemistry, 291 (8), 3759-3766. https://doi.org/10.1074/jbc.R114.635995

Figure 1 Chamber design, evaluation of flow properties and bioreactor setup. (a) CAD model (exploded view) and (b) photograph of the assembled culture chamber with $6 \mathrm{~mm}$ channel height and flow homogenizing insert. (c) Corresponding velocity streamline profile within the fluid channel and (d) surface shear stress obtained by CFD simulation at a volume flow rate of $6.4 \mathrm{~mL} \mathrm{~min}{ }^{-1}$. (e) Schematic illustration of the bioreactor circuit.

Figure 2 Morphology and proliferation of HT29-MTX cells under static and dynamic culture conditions. (a) Morphology was assessed via phase contrast microscopy and (b) newly synthesized DNA was visualized with EdU-Alexa ${ }^{(\mathrm{r})} 488$ (green) and imaged using confocal microscopy. Nuclei were counterstained with Hoechst 33342. (a) and (b) show independent samples. (scale bar $200 \mu \mathrm{m}$ ).

Figure 3 Topography of HT29-MTX cells under static and dynamic conditions after three and two weeks, respectively. (a) Confocal images of F-actin (red) and nuclei (blue) staining at different z-positions (bottom, middle, top) and representative orthogonal views below (the dotted line indicates the cutting plane). White arrow indicates a detached spheroid. (b) Corresponding 3-dimensional surface reconstructions of z-stacks of the epithelium, modelled byImageJ .

Figure 4 Qualitative and quantitative characterization of mucus produced by HT29-MTX cells under static and dynamic culture conditions at various time points. Microscopy images of (a) Alcian blue and (b) PAS stained cells showing neutral mucins in purple and acidic mucins in blue, respectively. (scale bars $200 \mu \mathrm{m}$ ) (c) Photometrically quantified amount of neutral mucins after PAS-incubation. Data presented as mean \pm SEM with respect to week one under static conditions. (d) Adherent mucus layer thickness determined under native conditions via confocal imaging.

Figure 5 Confocal immunofluorescence images of HT29-MTX cells after three weeks of static and two weeks of dynamic culture stained with antibodies for the membrane-bound mucin MUC1 and secreted mucins MUC2 and MUC5AC (green). Nuclei were counterstained with Hoechst 33342 (blue).

Figure 6 Colocalization and fiber-formation of secreted mucins MUC5AC and MUC5B. Confocal immunofluorescence images of HT29-MTX cells after two weeks of dynamic culture stained with antibodies for MUC5AC (red) and MUC5B (green). Nuclei were counterstained with Hoechst 33342 (blue). White arrow indicates an example of colocalization of both mucins in secretory vesicles.

Figure 7 Gene expression levels and differentiation of HT29-MTX cells under static and dynamic culture conditions at various time points. Relative mRNA levels of (a) GAPDH as metabolic indicator, ALPi as an enterocyte-marker and (b) corresponding intracellular ALP-activity. Relative mRNA levels of different (c) secreted and (d) transmembrane mucins. Data presented as mean \pm SEM and fold-change relative to samples after one week of culture under static conditions.

Figure 8 Influence of culture conditions on the barrier function of HT29-MTX cells. (a) Illustration of cells seeded onto the top or bottom of Transwell ${ }^{\circledR}$-insert membranes, termed as "normal" and "reverse", respectively. (b) Measured TEER values after one and two weeks of static and dynamic culture. Data presented as mean $\pm \mathrm{SEM}$. 

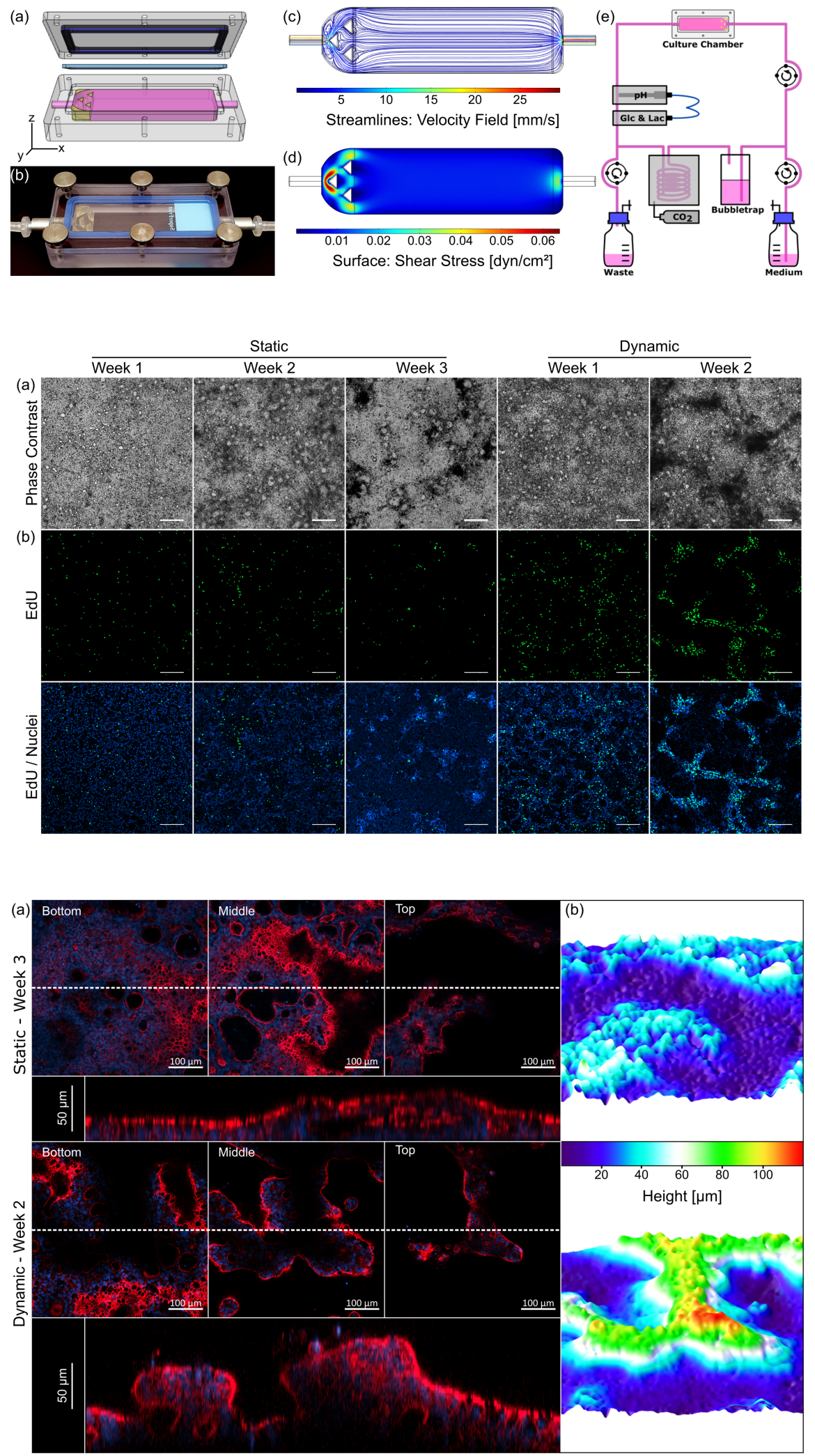

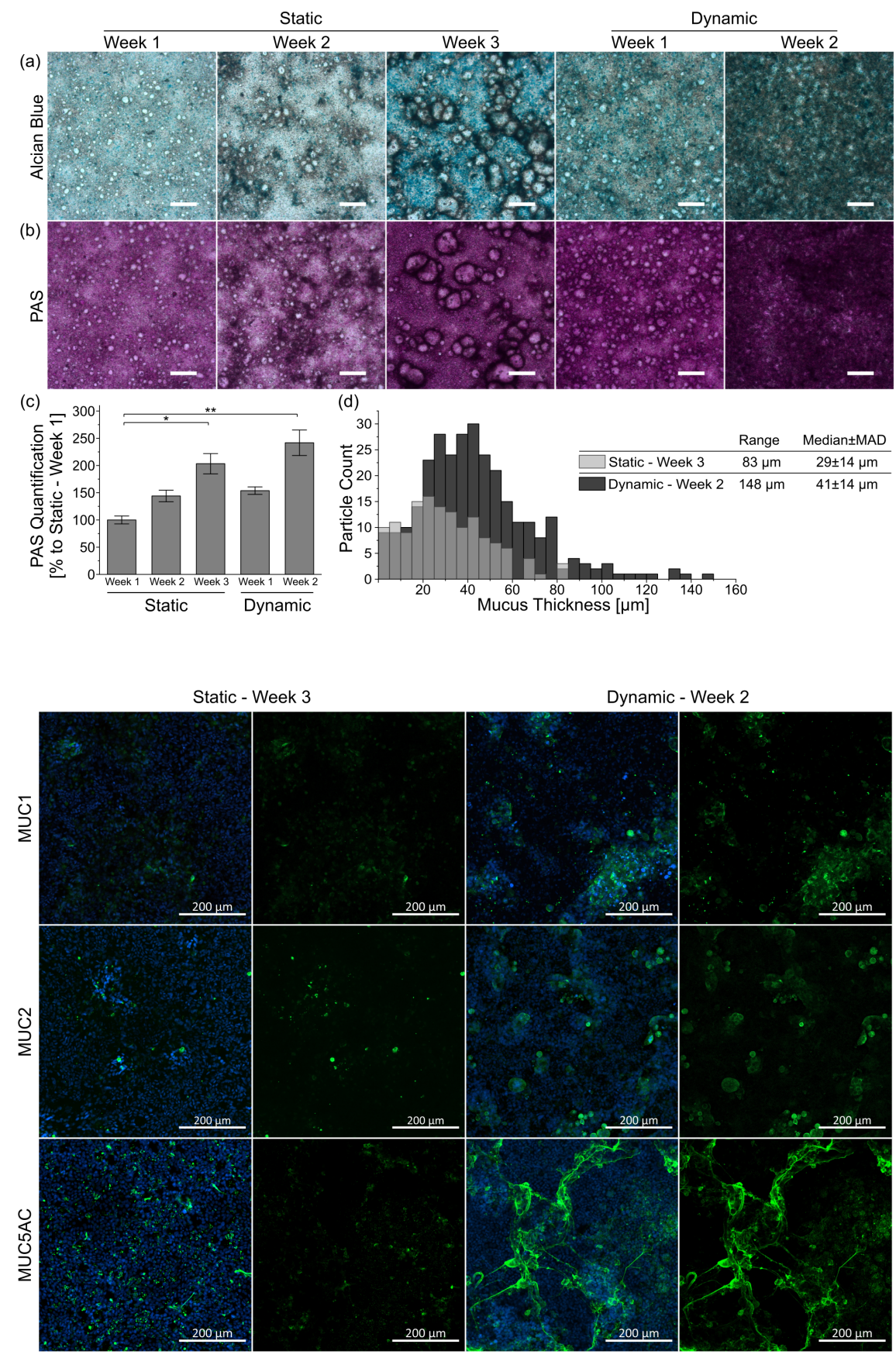

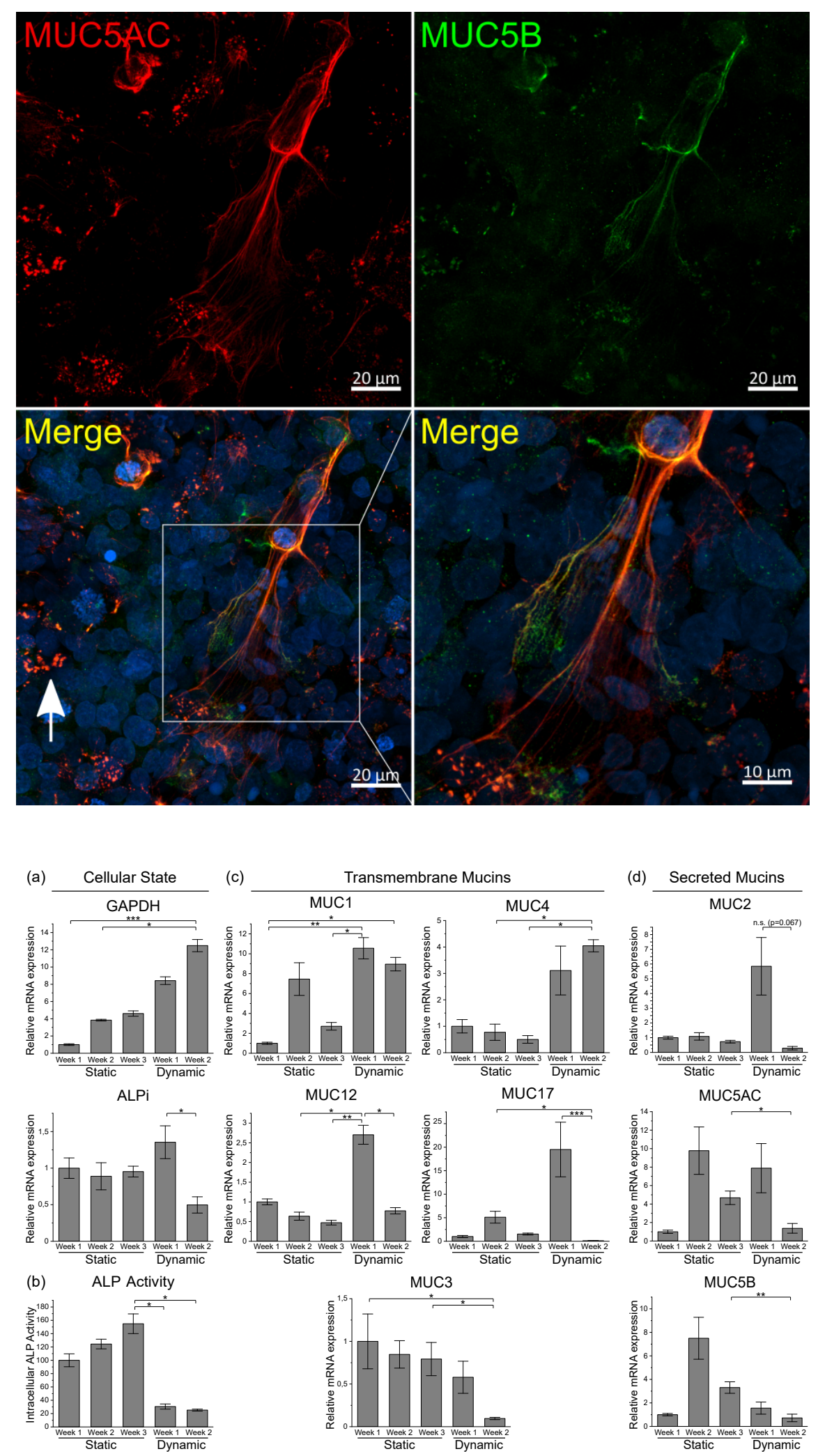

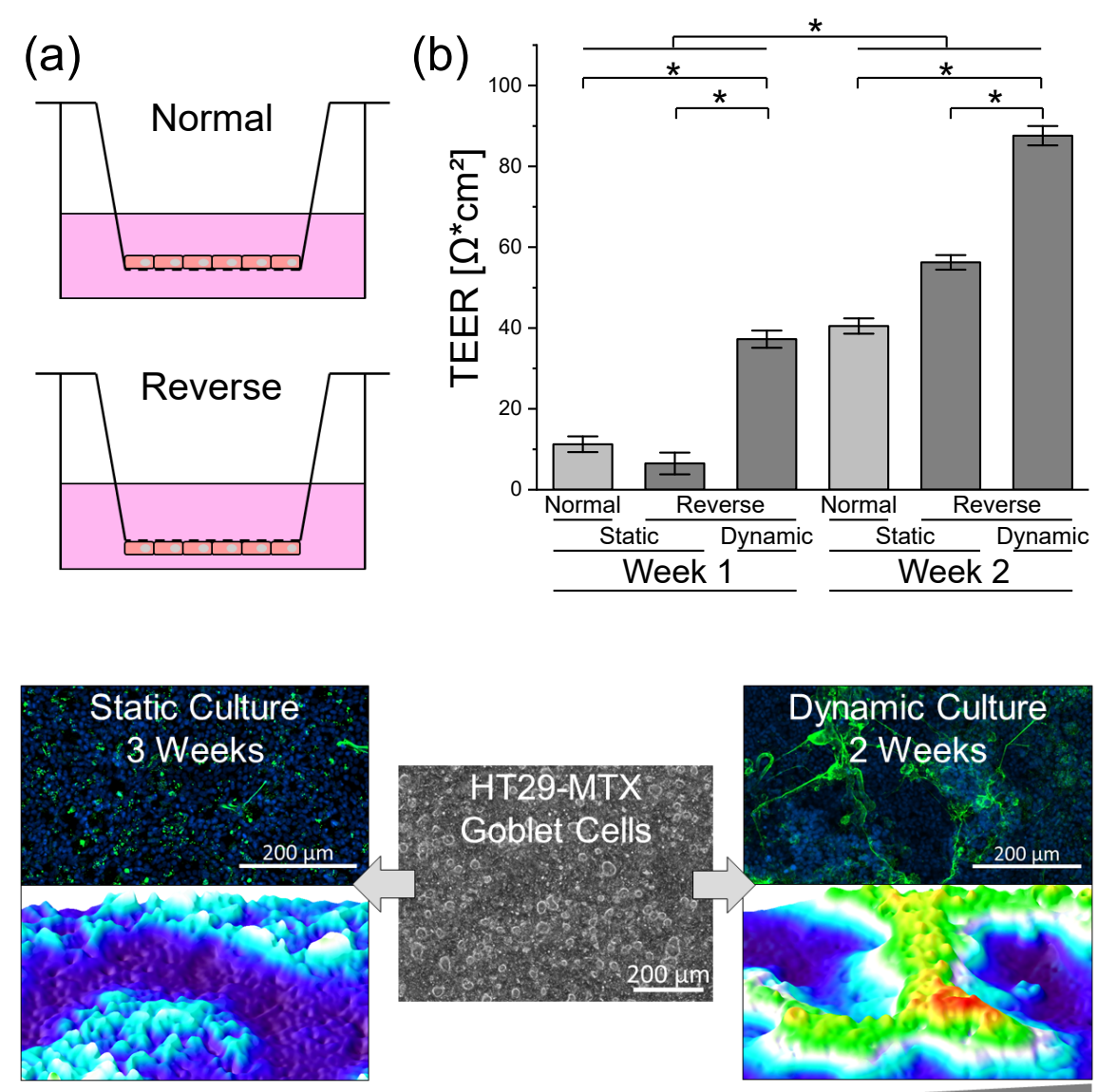

Differentiation • Mucus Production • Villi Formation 